\title{
A DELAY-DEPENDENT APPROACH TO ROBUST STABILITY FOR UNCERTAIN HYBRID BIDIRECTIONAL ASSOCIATIVE MEMORY NEURAL NETWORKS WITH TIME-VARYING DELAYS
}

\author{
Chien-Yu Lu \\ Department of Industrial Education and Technology, National Changhua University of Education, Changhua, Taiwan \\ 500, R.O.C
}

Koan-Yuh Chang

Department of Electronic Engineering, Chienkuo Technology University, Changhua 500, Taiwan, R.O.C

Hsun-Heng Tsai

Department of Biomechatronics Engineering, National Pingtung University of Science \& Technology, Pingtung, Taiwan 912, R.O.C.

Wen-Jer Chang

Department of Marine Engineering, National Taiwan Ocean University, Keelung 202, Taiwan, R.O.C, wjchang@mail.ntou.edu.tw

Follow this and additional works at: https://jmstt.ntou.edu.tw/journal

Part of the Electrical and Computer Engineering Commons

\section{Recommended Citation}

Lu, Chien-Yu; Chang, Koan-Yuh; Tsai, Hsun-Heng; and Chang, Wen-Jer (2010) "A DELAY-DEPENDENT APPROACH TO ROBUST STABILITY FOR UNCERTAIN HYBRID BIDIRECTIONAL ASSOCIATIVE MEMORY NEURAL NETWORKS WITH TIME-VARYING DELAYS," Journal of Marine Science and Technology. Vol. 18: Iss. 2, Article 2.

DOI: 10.51400/2709-6998.2315

Available at: https://jmstt.ntou.edu.tw/journal/vol18/iss2/2

This Research Article is brought to you for free and open access by Journal of Marine Science and Technology. It has been accepted for inclusion in Journal of Marine Science and Technology by an authorized editor of Journal of Marine Science and Technology. 


\title{
A DELAY-DEPENDENT APPROACH TO ROBUST STABILITY FOR UNCERTAIN HYBRID BIDIRECTIONAL ASSOCIATIVE MEMORY NEURAL NETWORKS WITH TIME-VARYING DELAYS
}

\author{
Chien-Yu Lu*, Koan-Yuh Chang**, Hsun-Heng Tsai***, and Wen-Jer Chang****
}

Key words: time-varying delays, linear matrix inequality, bidirectional associative memory neural networks, parameter uncertainty, robust stability.

\begin{abstract}
This paper performs a global robust stability analysis of a particular class of hybrid bidirectional associative memory time-varying delayed neural network with norm-bounded timevarying parameter uncertainties. The activation functions are assumed to be globally Lipschitz continuous. Globally delay-dependent robust stability criteria are derived in the form of linear matrix inequalities by introducing relaxation matrices which, when chosen properly, produce a less conservative result. Two numerical examples are given to illustrate the significant improvement obtained in the conservativeness of the delay bound.
\end{abstract}

\section{INTRODUCTION}

Bidirectional associative memory (BAM) neural networks were originally introduced in [18-20] and have been successfully deployed in pattern recognition and artificial intelligence applications [9, 14, 21, 23, 25]. The principal feature of BAM neural networks is the generalization of the single-layer autoassociative Hebbian correlator as a two-layered patternmatched heteroassociative circuit. Accordingly, BAM neural

Paper submitted 08/29/08; revised 02/26/09; accepted 04/13/09. Author for corresponding: Wen-Jer Chang (e-mail: wjchang@mail.ntou.edu.tw).

*Department of Industrial Education and Technology, National Changhua University of Education, Changhua, Taiwan 500, R.O.C.

**Department of Electronic Engineering, Chienkuo Technology University, Changhua 500, Taiwan, R.O.C.

***Department of Biomechatronics Engineering, National Pingtung University of Science \& Technology, Pingtung, Taiwan 912, R.O.C.

****Department of Marine Engineering, National Taiwan Ocean University, Keelung 202, Taiwan, R.O.C. networks have received significant attention in the literature [1, $10,17]$. It is well known that time delays are frequently encountered in the electronic implementation of artificial neural networks due to the finite switching speeds of the amplifiers and the inherent time required for communication will introduce time delays in the interaction between neurons [22]. Time delays introduce oscillation and instability in BAM neural networks. Therefore, the stability of time-delay BAM neural networks has been the subject of intensive theoretical and practical investigation [1, 7, 8, 12, 15, 16, 24, 27].

The studies cited above provide only pure-delay models. However, hybrid BAM neural networks with both instantaneous and delayed signaling have also been investigated. For example, the convergence dynamics of hybrid BAM neural networks with distributed delays were considered in [22], and a sufficient condition for their solvability was presented. Adopting a similar approach, [2] presented a sufficient condition for the existence, uniqueness and global asymptotic stability of the equilibrium point for hybrid BAM neural networks with distributed time delays. However, in both [22] and [2] the results are delay-independent, that is, they do not include any information relating to the magnitude of the distributed or discrete delays. It is known that delay-dependent conditions are generally less conservative than delay-independent conditions; particularly when the magnitude of the delay is small. Although delay-dependent results for BAM neural networks were developed in [15] and [24] using pure-delay models, the literature does not report on globally delaydependent robust stability criteria for hybrid BAM neural networks with time-varying delays and parameter uncertainties. The objective of the present study is to address this perceived gap in the literature.

Accordingly, this paper addresses the problem of attaining globally robust stability in uncertain hybrid BAM neural networks with time-varying delays. In the present analysis, the parameter uncertainties are assumed to be time-varying but norm-bounded. Combining the Lyapunov-Krasovskii functional with linear matrix inequality (LMI) techniques, globally 
delay-dependent robust stability conditions for uncertain hybrid BAM neural networks with time-varying delays, expressed in terms of quadratic forms of state and LMI, are derived. This study also introduce relaxation matrices which, when chosen properly, produce a less conservative result [13, 26]. The maximum bound for the time delays which ensure that the hybrid uncertain BAM neural network with timevarying delays achieves a globally robust stability is obtained by solving a quasi-convex optimization problem. Two numerical examples are provided to demonstrate the effectiveness of the method.

Throughout this paper, the notation $X \geq Y(X>Y)$ for symmetric matrices $X$ and $Y$ indicates that the matrix $X-Y$ is positive and semi-definite (respectively, positive definite), $Z^{T}$ represents the transpose of matrix $Z$, and the vector norm $\|\cdot\|$ indicates the Euclidean vector norm, that is $\|W\|=$ $\lambda_{M}^{1 / 2}\left(W^{T} W\right)$, where $\lambda_{M}(W)$ (respectively $\lambda_{m}(W)$ ) denotes the operation of taking the maximum (respectively, minimum) eigenvalue of $W$.

\section{SYSTEM DESCRIPTION AND PROBLEM FORMULATION}

Consider the following hybrid BAM neural network with a time-varying delay described by non-linear differential equations of the form [2]

$$
\begin{aligned}
\dot{u}_{i}(t)= & -\left(a_{i}+\Delta a_{i}(t)\right) u_{i}(t)+\sum_{j=1}^{m}\left(w_{i j}+\Delta w_{i j}(t)\right) g_{j}\left(z_{j}(t)\right) \\
& +\sum_{j=1}^{m}\left(w_{i j}^{v}+\Delta w_{i j}^{v}(t)\right) g_{j}\left(z_{j}(t-h(t))\right)+I_{i}, \\
& i=1,2, \cdots, n,
\end{aligned}
$$

and

$$
\begin{aligned}
\dot{z}_{j}(t)= & -\left(b_{j}+\Delta b_{j}(t)\right) z_{j}(t)+\sum_{i=1}^{n}\left(c_{j i}+\Delta c_{j i}(t)\right) g_{i}\left(u_{i}(t)\right) \\
& +\sum_{j=1}^{n}\left(c_{j i}^{v}+\Delta c_{j i}^{v}(t)\right) g_{i}\left(u_{i}(t-\tau(t))\right)+J_{j}, \\
& j=1,2, \cdots, m,
\end{aligned}
$$

with

$$
\begin{aligned}
u_{i}(t) & =\phi_{i}(t), \quad z_{j}(t)=\varphi_{j}(t), \quad t \in[-\max \{h, \tau\}, 0], \\
i & =1,2, \cdots, n, \quad j=1,2, \cdots, m .
\end{aligned}
$$

In the above, $u_{i}(t)$ and $z_{j}(t)$ denote the state of the $i$ th and $j$ th neurons at time $t$, respectively; $a_{i}$ and $b_{j}$ are the rate at which the $i$ th and $j$ th units reset their potential to the resting state in isolation, i.e. when disconnected from the network and the external inputs at time $t$, respectively; and $w_{i j}, w_{i j}^{v}, c_{j i}$ and $c_{j i}^{v}$ are synaptic connection strengths at the time $t, \Delta a_{i}(t), \Delta b_{j}(t)$, $\Delta w_{i j}(t), \Delta w_{i j}^{v}(t), \Delta c_{j i}(t)$ and $\Delta c_{j i}^{v}(t)$ denote unknown constants representing time-varying parameter uncertainties. Furthermore, $g_{i}$ and $g_{j}$ are the activation functions of the $i$ th and $j$ th neurons, respectively; and $I_{i}$ and $J_{j}$ are the external biases on the $i$ th and $j$ th neurons at time $t$. Finally, in (3), $\phi_{i}(t)$ and $\varphi_{j}(t)$ denote the initial conditions of the $i$ th and $j$ th neurons, respectively; $h(t)$ and $\tau(t)$ represent transmission delays which satisfy

$$
\begin{cases}0 \leq \tau(t) \leq \tau, & \dot{\tau}(t) \leq d_{\tau} \\ 0 \leq h(t) \leq h, & \dot{h}(t) \leq d_{h}\end{cases}
$$

Denote

$u(t)=\left[u_{1}(t), u_{2}(t), \cdots, u_{n}(t)\right]^{T}, z(t)=\left[z_{1}(t), z_{2}(t), \cdots, z_{m}(t)\right]^{T}$,

$$
\begin{gathered}
g(u(t))=\left[g_{1}\left(u_{1}(t), g_{2}\left(u_{2}(t)\right), \cdots, g_{n}\left(u_{n}(t)\right)\right]^{T},\right. \\
g(z(t))=\left[g_{1}\left(z_{1}(t), g_{2}\left(z_{2}(t)\right), \cdots, g_{m}\left(z_{m}(t)\right)\right]^{T},\right. \\
I=\left[I_{1}, I_{2}, \cdots, I_{n}\right]^{T}, J=\left[J_{1}, J_{2}, \cdots, J_{m}\right]^{T},
\end{gathered}
$$

$\phi(t)=\left[\phi_{1}(t), \phi_{2}(t), \cdots, \phi_{n}(t)\right]^{T}, \varphi(t)=\left[\varphi_{1}(t), \varphi_{2}(t), \cdots, \varphi_{m}(t)\right]^{T}$

Therefore, Eqs. (1) and (2) can be expressed as

$$
\begin{aligned}
\dot{u}(t)= & -(A+\Delta A(t)) u(t)+(W+\Delta W(t)) g(z(t)) \\
& +\left(W^{v}+\Delta W^{v}(t)\right) g(z(t-h(t)))+I, \\
\dot{z}(t)= & -(B+\Delta B(t)) z(t)+(C+\Delta C(t)) g(u(t)) \\
& +\left(C^{v}+\Delta C^{v}(t)\right) g(u(t-\tau(t)))+J,
\end{aligned}
$$

with

$$
u(t)=\phi(t), z(t)=\varphi(t), t \in[-\max \{h, \tau\}, 0],
$$

where

$A=\operatorname{diag}\left(a_{1}, a_{2}, \ldots, a_{n}\right), B=\operatorname{diag}\left(b_{1}, b_{2}, \ldots, b_{m}\right), W=\left[w_{i j}\right]_{n \times m}$,
$W^{v}=\left[w_{i j}^{v}\right]_{n \times m}, C=\left[c_{j i}\right]_{m \times n}$, and $C^{v}=\left[c_{j i}^{v}\right]_{m \times n}$.

Assume that $\Delta A(t), \Delta W(t), \Delta W^{v}(t), \Delta B(t), \Delta C(t)$ and $\Delta C^{v}(t)$ are of the form

$$
\begin{aligned}
& {\left[\begin{array}{l}
\Delta A(t) \Delta W(t) \Delta W^{v}(t) \Delta B(t) \Delta C(t) \Delta C^{v}(t)
\end{array}\right]} \\
& \quad=D F(t)\left[\begin{array}{llllll}
E_{1} & E_{2} & E_{3} & E_{4} & E_{5} & E_{6}
\end{array}\right]
\end{aligned}
$$


where $D, E_{1}, E_{2}, E_{3}, E_{4}, E_{5}$ and $E_{6}$ are known real constant matrices and $F(t)$ is an unknown real-valued time-varying matrix satisfying

$$
F^{T}(t) F(t) \leq I, \forall t
$$

Assume further that all the elements of $F(t)$ are Lebesgue measurable. $\Delta A(t), \Delta W(t), \Delta W^{v}(t), \Delta B(t), \Delta C(t)$ and $\Delta C^{v}(t)$ are said to be admissible if both (5) and (6) hold.

Throughout this paper it is assumed that the activation functions satisfy the following assumption.

Assumption 1 (Lipschitz condition) [2].

(A1) There exist positive constants $\alpha_{i}$ and $\beta_{j}, i=1,2, \cdots, n$ and $j=1,2, \cdots, m$ such that

$$
\begin{aligned}
& \left|g_{i}\left(\zeta_{1}\right)-g_{i}\left(\zeta_{2}\right)\right| \leq \alpha_{i}\left|\zeta_{1}-\zeta_{2}\right|, \zeta_{1} \neq \zeta_{2}, i=1, \cdots, n, \\
& \left|g_{j}\left(\xi_{1}\right)-g_{i}\left(\xi_{2}\right)\right| \leq \beta_{j}\left|\xi_{1}-\xi_{2}\right|, \xi_{1} \neq \xi_{2}, j=1, \cdots, m,
\end{aligned}
$$

for all $\zeta_{1}, \zeta_{2}, \xi_{1}$ and $\xi_{2} \in R$.

(A2) There exist positive constants $M_{i}, i=1, \ldots, n$ and $L_{i}, j=$ $1, \ldots, m$ such that $\left|g_{i}(u)\right| \leq M_{i}$ and $\left|g_{j}(z)\right| \leq L_{j}$ for all $u$, $z \in R$. Note that this assumption requires that the functions be bounded. Under assumption (A2), the hybrid BAM neural network defined by (5) and (6) always has an equilibrium point which can be found from various fixed point theorems, e.g. Brouwer's fixed point theorem, Schauder's fixed point theorem, or contraction mapping. In the following, this study analyzes the global asymptotic stability of the equilibrium point, which in turn implies the uniqueness of the equilibrium point.

To simplify the asymptotic stability analysis of (5) and (6), it is assumed that $u^{*}=\left[u_{1}^{*}, u_{2}^{*}, \cdots, u_{n}^{*}\right]^{T}$ and $z^{*}=$ $\left[z_{1}^{*}, z_{2}^{*}, \cdots, z_{m}^{*}\right]^{T}$ are the equilibrium points of the hybrid BAM neural network. The equilibrium points $u^{*}$ and $z^{*}$ are then shifted to the origin by letting $x(t)=u(t)-u^{*}, y(t)=z(t)-z^{*}$, $f(x(t))=g(u(t))-g\left(u^{*}\right)$ and $f(y(t))=g(z(t))-g\left(z^{*}\right)$. Consequently, the system given in (5) and (6) can be expressed as

$$
\begin{aligned}
\dot{x}(t)= & -(A+\Delta A(t)) x(t)+(W+\Delta W(t)) f(y(t)) \\
& +\left(W^{v}+\Delta W^{v}(t)\right) f(y(t-h(t))), \\
\dot{y}(t)= & -(B+\Delta B(t)) y(t)+(C+\Delta C(t)) f(x(t)) \\
& +\left(C^{v}+\Delta C^{v}(t)\right) f(x(t-\tau(t))),
\end{aligned}
$$

with

$$
u(t)=\phi(t), z(t)=\varphi(t), t \in[-\max \{h, \tau\}, 0],
$$

where

$$
\begin{gathered}
x(t)=\left[x_{1}(t), x_{2}(t), \cdots, x_{n}(t)\right]^{T}, y(t)=\left[y_{1}(t), y_{2}(t), \cdots, y_{m}(t)\right]^{T}, \\
f(x(t))=\left[f_{1}\left(x_{1}(t), f_{2}\left(x_{2}(t)\right), \cdots, f_{n}\left(x_{n}(t)\right)\right]^{T},\right. \\
f(y(t))=\left[f_{1}\left(y_{1}(t), f_{2}\left(y_{2}(t)\right), \cdots, f_{m}\left(y_{m}(t)\right)\right]^{T} .\right.
\end{gathered}
$$

Hence, it is easily shown that

$$
\begin{gathered}
\left|f_{i}\left(x_{i}\right)\right| \leq \alpha_{i}\left|x_{i}\right| \text {, and } f_{i}(0)=0, i=1, \cdots, n . \\
\left|f_{j}\left(y_{j}\right)\right| \leq \beta_{j}\left|y_{j}\right| \text {, and } f_{j}(0)=0, j=1, \cdots, m .
\end{gathered}
$$

Definition $1[5,11]$. The equilibrium point (trivial solution) 0 is said to be globally asymptotically stable if it is locally stable in the Lyapunov sense and is globally attractive, where global attractivity indicates that every trajectory tends to the equilibrium point as $t \rightarrow \infty$.

The objective of the present analysis is to establish the delay-dependent conditions which cause the hybrid uncertain BAM neural network with time-varying delays given in (12) and (13) to attain globally asymptotically robust stability. More specifically, for given scalars $\tau>0$ and $h>0$, the main aim of this study is to determine whether the system in (12) and (13) has globally asymptotically robust stability for all delays in the range $0 \leq \tau(t) \leq \tau$ and $0 \leq h(t) \leq h$.

\section{MATHEMATICAL FORMULATION OF THE PROPOSED APPROACH}

This section explores the globally robust stability for system given in (12) and (13). The analysis commences by using the LMI approach to develop some results which are essential to introduce the following Lemma 1 for the development of our main theorem.

Lemma 1 [3]. Let $A, D, S, F$ and $P$ be real matrices of appropriate dimensions with $P>0$ and $F$ satisfying $F^{T}(t) F(t) \leq I$. Then the following statements hold

(a) For any $\varepsilon>0$ and vectors $x, y \in R^{n}$

$$
2 x^{T} D F S y \leq \varepsilon^{-1} x^{T} D D^{T} x+\varepsilon y^{T} S^{T} S y,
$$

(b) For vectors $x, y \in R^{n}$

$$
2 x^{T} D S y \leq x^{T} D P D^{T} x+y^{T} S^{T} P^{-1} S y .
$$

For any matrices $Z_{i}, S_{i}, N_{i}$ and $M_{i}(i=1,2,3,4,5)$ of appropriate dimensions, it follows from null equations that $[13$, 26] 


$$
\begin{gathered}
{\left[x^{T}(t) Z_{1}+x^{T}(t-\tau(t)) Z_{2}+f^{T}(y(t)) Z_{3}+f^{T}(y(t-h(t))) Z_{4}\right.} \\
\left.+\dot{x}^{T}(t) Z_{5}\right] \times\left[x(t)-x(t-\tau(t))-\int_{t-\tau(t)}^{t} \dot{x}(s) d s\right]=0
\end{gathered}
$$

$$
\begin{gathered}
{\left[y^{T}(t) S_{1}+y^{T}(t-h(t)) S_{2}+f^{T}(x(t)) S_{3}+f^{T}(x(t-\tau(t))) S_{4}\right.} \\
\left.+\dot{y}^{T}(t) S_{5}\right] \times\left[y(t)-y(t-h(t))-\int_{t-h(t)}^{t} \dot{y}(s) d s\right]=0,
\end{gathered}
$$

$$
\begin{aligned}
& {\left[x^{T}(t) M_{1}+x^{T}(t-\tau(t)) M_{2}+f^{T}(y(t)) M_{3}+f^{T}(y(t-h(t))) M_{4}\right.} \\
& \left.\quad+\dot{x}^{T}(t) M_{5}\right] \times\{\dot{x}(t)+(A+\Delta A(t)) x(t)-(W+\Delta W(t)) f(y(t)) \\
& \left.\quad-\left(W_{1}+\Delta W_{1}(t)\right) f(y(t-h(t)))\right\}=0
\end{aligned}
$$

$$
\begin{aligned}
& {\left[y^{T}(t) N_{1}+y^{T}(t-h(t)) N_{2}+f^{T}(x(t)) N_{3}+f^{T}(x(t-\tau(t))) N_{4}\right.} \\
& \left.\quad+\dot{y}^{T}(t) N_{5}\right] \times\{\dot{y}(t)+(B+\Delta B(t)) x(t)-(C+\Delta C(t)) f(x(t)) \\
& \left.\quad-\left(C_{1}+\Delta C_{1}(t)\right) f(x(t-\tau(t)))\right\}=0 .
\end{aligned}
$$

To study the globally robust stable for the hybrid uncertain BAM neural network with time-varying delays, the following theorem reveals that such conditions can be expressed in terms of LMIs.

Theorem 1. Under the assumption 1 (A1), for any given $\tau>0$, $h>0$, satisfying $0 \leq \tau(t) \leq \tau, 0 \leq h(t) \leq h, \dot{\tau}(t) \leq d_{\tau}$ and $\dot{h}(t) \leq d_{h}$, (12) and (13) are globally robust stable if there exist matrices $P_{1}>0, P_{2}>0, Q_{1}>0, Q_{2}>0, R_{1}>0, R_{2}>0$, $T_{1}>0, T_{2}>0$ and matrices $Z_{i}, S_{i}, N_{i}$ and $M_{i}(i=1,2,3,4,5)$ of appropriate dimensions and positive scalars $\varepsilon_{1}$ and $\varepsilon_{2}$ such that the following linear matrix inequalities hold

$$
\begin{aligned}
& {\left[\begin{array}{cccc}
\Omega & M D & \varepsilon_{1} E^{T} & \tau \mathrm{Z} \\
D^{T} M^{T} & -\varepsilon_{1} & 0 & 0 \\
\varepsilon_{1} E & 0 & -\varepsilon_{1} & 0 \\
\tau Z^{T} & 0 & 0 & -\tau T_{1}
\end{array}\right]<0,} \\
& {\left[\begin{array}{cccc}
\Pi & N D & \varepsilon_{2} \bar{E}^{T} & h \mathrm{~S} \\
D^{T} N^{T} & -\varepsilon_{2} & 0 & 0 \\
\varepsilon_{2} \bar{E} & 0 & -\varepsilon_{2} & 0 \\
h S^{T} & 0 & 0 & -h T_{2}
\end{array}\right]<0,}
\end{aligned}
$$

where

$$
\begin{aligned}
& \Omega=\left[\begin{array}{lllll}
\Omega_{11} & \Omega_{12} & \Omega_{13} & \Omega_{14} & \Omega_{15} \\
\Omega_{12}^{T} & \Omega_{22} & \Omega_{23} & \Omega_{24} & \Omega_{25} \\
\Omega_{13}^{T} & \Omega_{23}^{T} & \Omega_{33} & \Omega_{34} & \Omega_{35} \\
\Omega_{14}^{T} & \Omega_{24}^{T} & \Omega_{34}^{T} & \Omega_{44} & \Omega_{45} \\
\Omega_{15}^{T} & \Omega_{25}^{T} & \Omega_{35}^{T} & \Omega_{45}^{T} & \Omega_{55}
\end{array}\right], \\
& \Omega_{11}=M_{1} A+A^{T} M_{1}^{T}+Q_{1}+Z_{1}+Z_{1}^{T}, \\
& \Omega_{12}=Z_{2}-Z_{1}+A^{T} M_{2}^{T}, \Omega_{13}=-M_{1} W+A^{T} M_{3}^{T}+Z_{3}, \\
& \Omega_{14}=Z_{4}-M_{1} W_{1}+A^{T} M_{4}^{T}, \Omega_{15}=P_{1}+M_{1}+A^{T} M_{5}^{T}+Z_{5}, \\
& \Omega_{22}=-\left(1-d_{\tau}\right) Q_{1}-Z_{2}-Z_{2}^{T}, \Omega_{23}=-M_{2} W-Z_{3}, \\
& \Omega_{24}=-M_{2} W_{1}-Z_{4}, \Omega_{25}=M_{2}-Z_{5}, \Omega_{33}=R_{2}-M_{3} W-W^{T} M_{3}^{T}, \\
& \Omega_{34}=-M_{3} W_{1}-W^{T} M_{4}^{T}, \Omega_{35}=M_{3}-W^{T} M_{5}^{T}, \\
& \Omega_{44}=-\left(1-d_{h}\right) R_{2}-M_{4} W_{1}-W_{1}^{T} M_{4}^{T}, \Omega_{45}=M_{4}-W_{1}^{T} M_{5}^{T}, \\
& \Omega_{55}=M_{5}+M_{5}^{T}+\tau T_{1},
\end{aligned}
$$$$
\Pi=\left[\begin{array}{lllll}
\Pi_{11} & \Pi_{12} & \Pi_{13} & \Pi_{14} & \Pi_{15} \\
\Pi_{12}^{T} & \Pi_{22} & \Pi_{23} & \Pi_{24} & \Pi_{25} \\
\Pi_{13}^{T} & \Pi_{23}^{T} & \Pi_{33} & \Pi_{34} & \Pi_{35} \\
\Pi_{14}^{T} & \Pi_{24}^{T} & \Pi_{34}^{T} & \Pi_{44} & \Pi_{45} \\
\Pi_{15}^{T} & \Pi_{25}^{T} & \Pi_{35}^{T} & \Pi_{45}^{T} & \Pi_{55}
\end{array}\right],
$$$$
\Pi_{11}=S_{1}+S_{1}^{T}+Q_{2}+N_{1} B+B^{T} N_{1}^{T}, \Pi_{12}=S_{2}-S_{1}+B^{T} N_{2}^{T},
$$$$
\Pi_{13}=-N_{1} C+B^{T} N_{3}^{T}+S_{3}, \Pi_{14}=S_{4}-N_{1} C_{1}+B^{T} N_{4}^{T},
$$$$
\Pi_{15}=P_{2}+N_{1}+B^{T} N_{5}^{T}+S_{5}, \Pi_{22}=-\left(1-d_{h}\right) Q_{2}-S_{2}-S_{2}^{T},
$$$$
\Pi_{23}=-N_{2} C-S_{3}, \Pi_{24}=-N_{2} C_{1}-S_{4}, \Pi_{25}=N_{2}-S_{5},
$$$$
\Pi_{33}=R_{1}-N_{3} C-C^{T} N_{3}^{T}, \Pi_{34}=-N_{3} C_{1}-C^{T} N_{4}^{T},
$$$$
\Pi_{35}=N_{3}-C^{T} N_{5}^{T}, \Pi_{44}=-\left(1-d_{\tau}\right) R_{1}-N_{4} C_{1}-C_{1}^{T} N_{4}^{T},
$$$$
\Pi_{45}=N_{4}-C_{1}^{T} N_{5}^{T}, \Pi_{55}=N_{5}+N_{5}^{T}+h T_{2},
$$$$
M=\left[\begin{array}{lllll}
M_{1}^{T} & M_{2}^{T} & M_{3}^{T} & M_{4}^{T} & M_{5}^{T}
\end{array}\right]^{T},
$$$$
Z=\left[\begin{array}{lllll}
Z_{1}^{T} & Z_{2}^{T} & Z_{3}^{T} & Z_{4}^{T} & Z_{5}^{T}
\end{array}\right]^{T},
$$ 
$E=\left[\begin{array}{lllll}E_{1} & 0 & -E_{2} & -E_{3} & 0\end{array}\right], \bar{E}=\left[\begin{array}{lllll}E_{4} & 0 & -E_{5} & -E_{6} & 0\end{array}\right]$,

$N=\left[\begin{array}{lllll}N_{1}^{T} & N_{2}^{T} & N_{3}^{T} & N_{4}^{T} & N_{5}^{T}\end{array}\right]^{T}$,

$S=\left[\begin{array}{lllll}S_{1}^{T} & S_{2}^{T} & S_{3}^{T} & S_{4}^{T} & S_{5}^{T}\end{array}\right]^{T}$.

Proof. Consider the following Lyapunov-Krasovskii functional for the system in (12) and (13)

$V(t)=V_{1}(t)+V_{2}(t)+V_{3}(t)+V_{4}(t)+V_{5}(t)+V_{6}(t)+V_{7}(t)+V_{8}(t)$,

where

$$
\begin{gathered}
V_{1}(t)=x^{T}(t) P_{1} x(t), \\
V_{2}(t)=y^{T}(t) P_{2} y(t), \\
V_{3}(t)=\int_{t-\tau(t)}^{t} x^{T}(\alpha) Q_{1} x(\alpha) d \alpha, \\
V_{4}(t)=\int_{t-h(t)}^{t} y^{T}(\alpha) Q_{2} y(\alpha) d \alpha, \\
V_{5}(t)=\int_{t-\tau(t)}^{t} f^{T}(x(\alpha)) R_{1} f(x(\alpha)) d \alpha, \\
V_{6}(t)=\int_{t-h(t)}^{t} f^{T}(y(\alpha)) R_{2} f(y(\alpha)) d \alpha, \\
V_{7}(t)=\int_{t-\tau}^{t} \int_{\alpha}^{t} \dot{x}^{T}(\theta) T_{1} \dot{x}(\theta) d \theta d \alpha, \\
V_{8}(t)=\int_{t-h}^{t} \int_{\alpha}^{t} \dot{y}^{T}(\theta) T_{2} \dot{y}(\theta) d \theta d \alpha .
\end{gathered}
$$

Taking the derivative of $V(t)$ with respect to $t$ along the trajectories of (12) and (13) yields

$$
\begin{aligned}
\dot{V}(t)= & 2 x^{T}(t) P_{1} \dot{x}(t)+2 y^{T}(t) P_{2} \dot{y}(t)+x^{T}(t) Q_{1} x(t) \\
& -(1-\dot{\tau}(t)) x^{T}(t-\tau(t)) Q_{1} x(t-\tau(t))+y^{T}(t) Q_{2} y(t) \\
& -(1-\dot{h}(t)) y^{T}(t-\tau(t)) Q_{2} y(t-\tau(t))+f^{T}(x(t)) R_{1} f(x(t)) \\
& -(1-\dot{\tau}(t)) f^{T}(x(t-\tau(t))) R_{1} f(x(t-\tau(t)))
\end{aligned}
$$

$$
\begin{aligned}
& +f^{T}(y(t)) R_{2} f(y(t))-(1-\dot{h}(t)) f^{T}(y(t-h(t))) R_{2} f(y(t-h(t))) \\
& +\tau \dot{x}^{T}(t) T_{1} \dot{x}(t)-\int_{t-\tau}^{t} \dot{x}^{T}(\alpha) T_{1} \dot{x}(\alpha) d \alpha \\
& +h \dot{y}^{T}(t) T_{2} \dot{y}(t)-\int_{t-h}^{t} \dot{y}^{T}(\alpha) T_{2} \dot{y}(\alpha) d \alpha .
\end{aligned}
$$

Substituting (17)-(20) into (30) yields

$\dot{V}(t) \leq 2 x^{T}(t) P_{1} \dot{x}(t)+2 y^{T}(t) P_{2} \dot{y}(t)+x^{T}(t) Q_{1} x(t)$

$$
\begin{aligned}
& -\left(1-d_{x}\right) x^{T}(t-\tau(t)) Q_{1} x(t-\tau(t))+y^{T}(t) Q_{2} y(t) \\
& -\left(1-d_{h}(t)\right) y^{T}(t-\tau(t)) Q_{2} y(t-\tau(t))+f^{T}(x(t)) R_{1} f(x(t)) \\
& -\left(1-d_{x}(t)\right) f^{T}(x(t-\tau(t))) R_{1} f(x(t-\tau(t)))+f^{T}(y(t)) R_{2} f(y(t)) \\
& -\left(1-d_{h}(t)\right) f^{T}(y(t-h(t))) R_{2} f(y(t-h(t))) \\
& +\tau \dot{x}^{T}(t) T_{1} \dot{x}(t)-\int_{t-\tau}^{t} \dot{x}^{T}(\alpha) T_{1} \dot{x}(\alpha) d \alpha \\
& +h \dot{y}^{T}(t) T_{2} \dot{y}(t)-\int_{t-h}^{t} \dot{y}^{T}(\alpha) T_{2} \dot{y}(\alpha) d \alpha \\
& +2\left[x^{T}(t) Z_{1}+x^{T}(t-\tau(t)) Z_{2}+f^{T}(y(t)) X_{3}+f^{T}(y(t-h(t))) Z_{4}\right. \\
& \left.+\dot{x}^{T}(t) Z_{5}\right] \times\left[x(t)-x(t-\tau(t))-\int_{t-\tau(t)}^{t} \dot{x}(\alpha) d \alpha\right] \\
& +2\left[y^{T}(t) S_{1}+y^{T}(t-h(t)) S_{2}+f^{T}(x(t)) S_{3}+f^{T}(x(t-\tau(t))) S_{4}\right. \\
& \left.+\dot{y}^{T}(t) S_{5}\right] \times\left[y(t)-y(t-h(t))-\int_{t-h(t)}^{t} \dot{y}(\alpha) d \alpha\right] \\
& +2\left[x^{T}(t) M_{1}+x^{T}(t-\tau(t)) M_{2}+f^{T}(x(t)) M_{3}+f^{T}(x(t-\tau(t))) M_{4}\right. \\
& \left.+\dot{x}^{T}(t) M_{5}\right] \times\{\dot{x}(t)+(A+\Delta A(t)) x(t)-(W+\Delta W(t)) f(y(t)) \\
& \left.-\left(W_{1}+\Delta W_{1}(t)\right) f(y(t-h(t)))\right\} \\
& +2\left[y^{T}(t) N_{1}+y^{T}(t-h(t)) N_{2}+f^{T}(x(t)) N_{3}+f^{T}(x(t-\tau(t))) N_{4}\right. \\
& \left.+\dot{y}^{T}(t) N_{5}\right] \times\{\dot{y}(t)+(B+\Delta B(t)) y(t)-(C+\Delta C(t)) f(x(t)) \\
& \left.-\left(C_{1}+\Delta C_{1}(t)\right) f(x(t-\tau(t)))\right\}
\end{aligned}
$$


From Lemma 1, it can be shown that

$$
\begin{aligned}
& -2\left[x^{T}(t) Z_{1}+x^{T}(t-\tau(t)) Z_{2}+f^{T}(y(t)) Z_{3}\right. \\
& \left.+f^{T}(y(t-h(t))) Z_{4}+\dot{x}^{T}(t) Z_{5}\right] \int_{t-\tau(t)}^{t} \dot{x}(\alpha) d \alpha \\
& \leq \tau q_{1}^{T}(t) Z T_{1}^{-1} Z^{T} q_{1}(t)+\int_{t-\tau(t)}^{t} \dot{x}^{T}(\alpha) T_{1} \dot{x}(\alpha) d \alpha, \\
& -2\left[y^{T}(t) S_{1}+y^{T}(t-h(t)) S_{2}+f^{T}(x(t)) S_{3}\right. \\
& \left.\quad+f^{T}(x(t-\tau(t))) S_{4}+\dot{y}^{T}(t) S_{5}\right] \int_{t-h(t)}^{t} \dot{y}(\alpha) d \alpha \\
& \quad \leq h q_{2}^{T}(t) S T_{2}^{-1} S^{T} q_{2}(t)+\int_{t-h(t)}^{t} \dot{y}^{T}(\alpha) T_{2} \dot{y}(\alpha) d \alpha,
\end{aligned}
$$

$$
\begin{aligned}
2[ & x^{T}(t) M_{1}+x^{T}(t-\tau(t)) M_{2}+f^{T}(x(t)) M_{3}+f^{T}(x(t-\tau(t))) M_{4} \\
& \left.+\dot{x}^{T}(t) M_{5}\right] \times\{\dot{x}(t)+(A+\Delta A(t)) x(t)-(W+\Delta W(t)) f(y(t)) \\
& \left.-\left(W_{1}+\Delta W_{1}(t)\right) f(y(t-h(t)))\right\} \leq 2 q_{1}^{T}(t) M(\bar{A}+D F(t) E) \\
& \leq 2 q_{1}^{T}(t) M \bar{A} q_{1}(t)+q_{1}^{T}(t) \varepsilon_{1}^{-1} M D D^{T} M^{T} q_{1}(t) \\
& +q_{1}^{T}(t) \varepsilon_{1} E^{T} E q_{1}(t),
\end{aligned}
$$

$$
\begin{aligned}
& 2\left[y^{T}(t) N_{1}+y^{T}(t-h(t)) N_{2}+f^{T}(x(t)) N_{3}+f^{T}(x(t-\tau(t))) N_{4}\right. \\
& \left.\quad+\dot{y}^{T}(t) N_{5}\right] \times\{\dot{y}(t)+(B+\Delta B(t)) y(t)-(C+\Delta C(t)) f(x(t)) \\
& \left.\quad-\left(C_{1}+\Delta C_{1}(t)\right) f(x(t-\tau(t)))\right\} \leq 2 q_{2}^{T}(t) N(\bar{B}+D F(t) \bar{E}) \\
& \quad \leq 2 q_{2}^{T}(t) N \bar{B} q_{2}(t)+q_{2}^{T}(t) \varepsilon_{2}^{-1} N D D^{T} N^{T} q_{2}(t) \\
& \quad+q_{2}^{T}(t) \varepsilon_{2} \bar{E}^{T} \bar{E} q_{2}(t)
\end{aligned}
$$

where

$$
\begin{aligned}
& q_{1}(t)=\left[\begin{array}{llllll}
x^{T}(t) & x^{T}(t-\tau(t)) & f^{T}(y(t)) & f^{T}(y(t-h(t))) & \dot{x}^{T}(t)
\end{array}\right]^{T}, \\
& q_{2}(t)=\left[\begin{array}{lllllll}
y^{T}(t) & y^{T}(t-h(t)) & f^{T}(x(t)) & f^{T}(x(t-\tau(t))) & \dot{y}^{T}(t)
\end{array}\right]^{T}, \\
& Z=\left[\begin{array}{lllllll}
Z_{1}^{T} & Z_{2}^{T} & Z_{3}^{T} & Z_{4}^{T} & Z_{5}^{T}
\end{array}\right]^{T}, S=\left[\begin{array}{llllll}
S_{1}^{T} & S_{2}^{T} & S_{3}^{T} & S_{4}^{T} & S_{5}^{T}
\end{array}\right]^{T}, \\
& M=\left[\begin{array}{llllll}
M_{1}^{T} & M_{2}^{T} & M_{3}^{T} & M_{4}^{T} & M_{5}^{T}
\end{array}\right]^{T}, \\
& N=\left[\begin{array}{llllll}
N_{1}^{T} & N_{2}^{T} & N_{3}^{T} & N_{4}^{T} & N_{5}^{T}
\end{array}\right]^{T}, \\
& \bar{A}=\left[\begin{array}{llllllll}
A & 0 & -W & -W_{1} & I
\end{array}\right], \bar{B}=\left[\begin{array}{llllll}
B & 0 & -C & -C_{1} & I
\end{array}\right],
\end{aligned}
$$

$$
E=\left[\begin{array}{lllll}
E_{1} & 0 & -E_{2} & -E_{3} & 0
\end{array}\right], \bar{E}=\left[\begin{array}{lllll}
E_{4} & 0 & -E_{5} & -E_{6} & 0
\end{array}\right] .
$$

Combining Eqs. (31)-(35) yields

$$
\dot{V}(t) \leq q_{1}^{T}(t) \Omega_{1} q_{1}(t)+q_{2}^{T}(t) \Pi_{1} q_{2}(t)
$$

where

$$
\begin{aligned}
& \Omega_{1}=\Omega+\tau Z T_{1}^{-1} Z^{T}+\varepsilon_{1}^{-1} M D D^{T} M^{T}+\varepsilon_{1} E^{T} E \\
& \Pi_{1}=\Pi+h S T_{2}^{-1} S^{T}+\varepsilon_{2}^{-1} N D D^{T} N^{T}+\varepsilon_{2} \bar{E}^{T} \bar{E}
\end{aligned}
$$

From (18) and (19) and using the well-known Schur complement [5], it can be shown that $\dot{V}(t)<0$. Therefore, it can be concluded from the Lyapunov-Krasovskii functional that the hybrid uncertain BAM neural network expressed in (12)(13) attains globally asymptotically robust stability. This completes the proof of Theorem 1.

Remark 1. Theorem 1 provides a sufficient condition for the globally asymptotically robust stability for system given in (12)-(13) and proposes a delay-dependent stability criterion. Recently the results have been studied in $[2,22]$ and are delay-independent stability criteria. Since, it is less conservative than a delay-independent condition in [2, 22]. Moreover, parameter uncertainties have also not been fully investigated in $[2,22]$. Also, the present results in [15] ignore some useful terms. This may bring conservativeness. In Theorem 1, all terms are reserved, which can reduce conservativeness to keep the stability of systems. In [15], the system is pure-delay model with constant time delay. Therefore, the results obtained in Theorem 1 for uncertain hybrid BAM neural networks with time-varying delays are more general than that presented in the literature [15].

Remark 2: In many cases, the derivative of time-varying delay $h(t)$ and $\tau(t)$ is known and may be small. Relaxation matrices are introduced into Theorem 1 , so, $d_{h}$ and $d_{\tau}$ can be any value or unknown due to $\Pi_{22}=-\left(1-d_{h}\right) Q_{2}-S_{2}-S_{2}^{T}$, $\Omega_{22}=-\left(1-d_{\tau}\right) Q_{1}-Z_{2}-Z_{2}^{T}$, where $Z_{2}, S_{2}$ are relaxation matrices with appropriate dimensions. The traditional assumption that derivatives of the delays are less than 1 is no longer required in our analysis. Therefore, Theorem 1 is applicable to more general and practical than the one investigated in [2] and [22].

Two numerical examples are now presented to demonstrate the usefulness of the proposed approach.

\section{NUMERICAL EXAMPLES}

Example 1: Consider the following hybrid uncertain BAM delayed neural network 


$$
\begin{aligned}
\dot{u}(t)= & -(A+\Delta A(t)) u(t)+(W+\Delta W(t)) g(z(t)) \\
& +\left(W^{v}+\Delta W^{v}(t)\right) g(z(t-h(t)))+I, \\
\dot{z}(t)= & -(B+\Delta B(t)) z(t)+(C+\Delta C(t)) g(u(t)) \\
& +\left(C^{v}+\Delta C^{v}(t)\right) g(u(t-\tau(t)))+J,
\end{aligned}
$$

where

$$
\begin{aligned}
& A=\left[\begin{array}{ll}
2 & 0 \\
0 & 0.9
\end{array}\right], W=\left[\begin{array}{ll}
-1 & 0 \\
-0.5 & -1
\end{array}\right], W_{1}=\left[\begin{array}{ll}
0.9 & -1.2 \\
0.05 & -0.9
\end{array}\right], \\
& D=\left[\begin{array}{ll}
0.1 & 0 \\
0 & 0.3
\end{array}\right], B=\left[\begin{array}{ll}
1.2 & 0 \\
0 & 1.1
\end{array}\right], \\
& C=\left[\begin{array}{ll}
0.3 & 0.2 \\
0.5 & 0.4
\end{array}\right], C_{1}=\left[\begin{array}{ll}
0.7 & 0.7 \\
0.5 & 1.3
\end{array}\right], \\
& E_{1}=\left[\begin{array}{ll}
0.02 & 0.02 \\
-0.1 & 0.2
\end{array}\right], E_{2}=\left[\begin{array}{ll}
-0.07 & 0.3 \\
-0.01 & 0.2
\end{array}\right], E_{3}=\left[\begin{array}{ll}
0.02 & -0.02 \\
0.1 & -0.1
\end{array}\right], \\
& E_{4}=\left[\begin{array}{ll}
0.01 & 0.02 \\
-0.1 & 0.3
\end{array}\right], E_{5}=\left[\begin{array}{ll}
-0.04 & 0.2 \\
-0.01 & 0.1
\end{array}\right], E_{6}=\left[\begin{array}{ll}
0.01 & -0.02 \\
0.2 & -0.1
\end{array}\right] .
\end{aligned}
$$

Utilizing Theorem 1 and $F^{T}(t) F(t) \leq I$, it is found that LMI equations (21) and (22) are feasible for any arbitrarily large $\tau$ and $h$ for different $d_{\tau}$ and $d_{h}$ (provided that the numerical computation is reliable). Therefore, in view of Theorem 1, the current hybrid uncertain BAM neural network attains globally robust stability in the sense of Definition 1.

Example 2: Consider the following uncertain BAM delayed neural network

$$
\dot{u}(t)=-(A+\Delta A(t)) u(t)+\left(W^{v}+\Delta W^{v}(t)\right) g(z(t-h(t)))+I,
$$

$$
\dot{z}(t)=-(B+\Delta B(t)) z(t)+\left(C^{v}+\Delta C^{v}(t)\right) g(u(t-\tau(t)))+J,
$$

$$
A=\left[\begin{array}{lll}
1 & 0 & 0 \\
0 & 1 & 0 \\
0 & 0 & 1
\end{array}\right], B=\left[\begin{array}{lll}
4 & 0 & 0 \\
0 & 4 & 0 \\
0 & 0 & 4
\end{array}\right], W^{v}=\left[\begin{array}{lll}
0.05 & 0.25 & 0.05 \\
0.10 & 0.05 & 0.15 \\
0.15 & 0.15 & 0.05
\end{array}\right]
$$$$
C^{v}=\left[\begin{array}{lll}
0.75 & 0.75 & 0.95 \\
0 & 0.50 & 0.75 \\
0.15 & 0.95 & 0.95
\end{array}\right], D=\left[\begin{array}{lll}
0.1 & 0 & 0.1 \\
0 & 0.3 & 0.1 \\
0 & 0.1 & 0.2
\end{array}\right]
$$

$$
\begin{aligned}
& E_{1}=\left[\begin{array}{cll}
0.02 & 0.02 & 0 \\
-0.1 & 0.1 & 0.1 \\
0.1 & 0 & 0.1
\end{array}\right], E_{3}=\left[\begin{array}{lll}
0.02 & -0.02 & 0 \\
0.1 & -0.1 & 0 \\
0.1 & 0 & 0.1
\end{array}\right], \\
& E_{4}=\left[\begin{array}{lll}
0.01 & 0.02 & 0 \\
-0.1 & 0.3 & 0.1 \\
0.1 & 0 & 0.2
\end{array}\right], E_{6}=\left[\begin{array}{ccl}
0.01 & -0.02 & 0 \\
0.2 & -0.1 & 0 \\
0.1 & 0.2 & 0.1
\end{array}\right] .
\end{aligned}
$$

All uncertain terms are zero in (39) and (40), it can be verified that Theorem 1 in [24] is feasible for all delays $0 \leq \sigma \leq$ 0.5 and $0 \leq \tau \leq 0.78$, Corollary 2 in [13] is feasible for all delays $0 \leq \tau \leq 1$ and $0 \leq \sigma \leq 0.5$. Also, Theorem 3.1 in [8] does not satisfy globally delay-dependent conditions for all delays $\tau$ and $\delta$ when $N$ and $M$ are zero. Theorem 1 in this paper is feasible for all delays $0 \leq \tau(t) \leq 3.78$ and $0 \leq h(t) \leq 3.78$. Therefore, Theorem 1 in this paper is less conservative than those in $[8,15,24]$ for delayed BAM neural network. Moreover, it can be checked that the delay-dependent robust stability conditions in $[1,2,22]$ is not satisfied. Therefore, they fail to conclude that uncertain hybrid BAM neural network with time-varying delays is globally delay-dependent robust stability. By resorting to Theorem 1 in this paper, it is found that LMIs in (21) and (22) are feasible for all delays satisfying $0 \leq \tau(t) \leq 10.58$ and $0 \leq h(t) \leq 10.58$. The solutions of Theorem 1 are as follows

$P_{1}=\left[\begin{array}{rrr}2.3061 & -0.2657 & -0.4443 \\ -0.2657 & 2.5420 & -0.3180 \\ -0.4443 & -0.3180 & 2.4346\end{array}\right]$

$P_{2}=\left[\begin{array}{rrr}1.4861 & -0.4376 & -0.9482 \\ -0.4376 & 2.6898 & -1.0997 \\ -0.9482 & -1.0997 & 1.9763\end{array}\right]$

$Q_{1}=\left[\begin{array}{rrr}1.5769 & -0.1885 & -0.3018 \\ -0.1885 & 1.7086 & -0.2005 \\ -0.3018 & -0.2005 & 1.6656\end{array}\right]$

$Q_{2}=\left[\begin{array}{rrr}1.6066 & -0.4482 & -0.7091 \\ -0.4482 & 1.9974 & -0.4595 \\ -0.7091 & -0.4595 & 1.6406\end{array}\right]$

$R_{1}=\left[\begin{array}{rrr}2.6751 & -0.0527 & 0.0321 \\ -0.0527 & 2.4971 & 0.5400 \\ 0.0321 & 0.5400 & 2.3424\end{array}\right]$, 


$$
\begin{aligned}
& R_{2}=\left[\begin{array}{lrr}
2.5036 & 0.3361 & 0.4186 \\
0.3361 & 2.9811 & 0.2920 \\
0.4186 & 0.2920 & 2.3636
\end{array}\right], \\
& T_{1}=\left[\begin{array}{rrr}
0.1745 & -0.0219 & -0.0403 \\
-0.0219 & 0.1960 & -0.0334 \\
-0.0403 & -0.0334 & 0.1843
\end{array}\right], \\
& T_{2}=\left[\begin{array}{rrr}
0.0261 & -0.0055 & -0.0203 \\
-0.0055 & 0.0659 & -0.0341 \\
-0.0203 & -0.0341 & 0.0453
\end{array}\right], \\
& \mathcal{E}_{1}=1.5575, \varepsilon_{2}=1.4104 .
\end{aligned}
$$

Thus, by Theorem 1, Eqs. (39) and (40) attains globally delaydependent robust stability. This shows that the condition given in Theorem 1 is less conservative than those in $[1,2,22]$ when checking the globally delay-dependent robust stability for a given uncertain hybrid BAM neural network with timevarying delays.

\section{CONCLUSIONS}

This study has investigated the problem of globally delay-dependent robust stability for a particular class of uncertain hybrid BAM neural network with time-varying delays. A sufficient condition for the solvability of this problem, which depends on the magnitude of the time delay, has been derived using the Lyapunov-Krasovskii functional and the LMI approach. It has been shown that the hybrid uncertain BAM with time-varying delays attains globally delay-dependent robust stability can be obtained by solving a quasi-convex optimization problem. Two numerical examples have been presented to demonstrate the effectiveness of the proposed approach.

\section{REFERENCES}

1. Arik, S. "Global asymptotic stability analysis of bidirectional associative memory neural networks with time delays," IEEE Transactions on Neural Networks, Vol. 16, pp. 580-586 (2005).

2. Arik, S. "Global asymptotic stability of hybrid bidirectional associative memory neural networks with time delays," Physics Letters A, Vol. 351, pp. 85-91 (2006).

3. Boukas, E. K. and Liu, Z. K., Deterministic and Stochastic Time Delay Systems, Birkhauser, Boston (2002).

4. Boyd, S., Ghaoui, L. Ei., Feron, E., and Balakrishnan, V., Linear Matrix Inequalities in System and Control Theory, SIAM, Philadelphia (1994).

5. Burton, T., Stability and Periodic Solution of Ordinary Differential Equations and Functional Differential Equations, Fl, Academic Press, Orlando (1985).

6. Cao, J. and Wang, L., "Periodic oscillatory solution of bidirectional as- sociative memory networks," Physical Review E, Vol. 61, pp. 1825-1828 (2000).

7. Cao, J. and Wang, L., "Exponential stability and periodic oscillatory solution in BAM networks with delays," IEEE Transactions on Neural Networks, Vol. 13, pp. 457-463 (2002).

8. Ding, K., Huang, N. J. and Xu, X., "Global robust exponential stability of interval general BAM neural network with mixed delays under uncertainty," Neural Processing Letters, Vol. 25, 127-141 (2007).

9. Gopalsamy, K. and He, X. Z., "Delay-independent stability in bidirectional associative memory networks," IEEE Transactions on Neural Networks, Vol. 5, pp. 998-1002 (1994).

10. Guo, S. J., Huang, L. H., B. X. Dai, B. X. and Zhang, Z. Z., "Global existence of periodic solution of BAM neural networks with variable coefficients," Phsics Letters A, Vol. 317, pp. 97-106 (2003).

11. Hale, J. K., Theory of Functional Differential Equations, Springer-Verlag, New York (1977)

12. Hari Rao V. Sree and Phaneendra, Bh. R. M., "Global dynamics of bidirectional associative memory neural networks involving transmission delays and dead zone," Neural Networks, Vol. 12, pp. 455-465 (1999).

13. He, Y., Wu, M., She, J. H., and Liu, G. P., "Parameter-dependent Lyapunov functional for stability of time-delay systems with polytopictype uncertainties," IEEE Transaction on Automatic Control, Vol. 49, pp. 828-832 (2004).

14. Hopfield, J. J., "Neurons with graded response have collective computational properties like those of two-state neurons," Proceedings of the National Academy of Sciences, Vol. 81, pp. 3088-3092 (1984).

15. Huang, X., Cao, J., and Huang, D., "LMI-based approach for delaydependent exponential stability analysis of BAM neural networks," Chaos, Solitons and Fractals, Vol. 24, pp. 885-898 (2005).

16. Kleinfeld, D., "Sequential state generation by model neural networks," Proceedings of the National Academy of Sciences, USA, Vol. 83, 94699473 (1986).

17. Kohonen, T., Self-Organization and Associative Memory, Springer, New York (1988).

18. Kosko, B., "Adaptive bidirectional associative memories," Applied Optics, Vol. 26, pp. 4947-4960 (1987).

19. Kosko, B., "Bidirectional associative memories," IEEE Transactions on Systems, Man, and Cybernetics, Vol. 18, pp. 49-60 (1988).

20. Kosko, B., A Dynamical System Approach Machine Intelligence, Prentice-Hall, Englewood Cliffs (1992).

21. Liang, J. and Cao, J., "Global asymptotic stability of bi-directional associative memory networks with distributed delays," Applied Mathematics and Computation, Vol. 152, pp. 415-424 (2004).

22. Liao, X., Wong, K., and Yang, S., "Convergence dynamics of hybrid bidirectional associative memory neural networks with distributed delays," Physics Letters A, Vol. 316, pp. 55-64 (2003).

23. Liao, X. and Yu, J., "Qualitative analysis of bidirectional associative memory with time delay," International Journal of Circuit Theory and Applications, Vol. 26, pp. 219-229 (1998).

24. Park, J. H., "A novel criterion for global asymptotic stability of BAM neural networks with time delays," Chaos, Solitons and Fractals, Vol. 29, 446-453 (2006).

25. Sree Hari Rao, V., Phaneendra Bh, R. M., and Prameela, V., "Global dynamics of bidirectional associative memory networks with transmission delays," Differential Equations and Dynamical Systems, Vol. 4, pp. 453-471 (1996).

26. Yue, D., Han, Q. L., and Peng, C., "State feedback controller design of network control systems," IEEE Transactions on Circuits and Systems-II: Express Briefs, Vol. 51, pp. 640-644 (2004).

27. Zhang J. and Yang, Y., Global stability analysis of bidirectional associative memory neural networks with time delay, International Journal of Circuit Theory and Applications, Vol. 29, pp. 185-196 (2001). 